\title{
Optimal visualization of heart vessels before percutaneous mitral annuloplasty
}

\author{
Rafał Młynarski1,2, Agnieszka Młynarska ${ }^{1,2}$, Jacek Wilczek ${ }^{1}$, Maciej Sosnowski ${ }^{2,3}$ \\ ${ }^{1}$ Department of Electrocardiology, Upper Silesian Medical Center, Katowice, Poland \\ ${ }^{2}$ Unit of Noninvasive Cardiovascular Diagnostics, Upper Silesian Medical Center, Katowice, Poland \\ ${ }^{3} 3^{\text {rd }}$ Division of Cardiology, Medical University of Silesia, Katowice, Poland
}

\begin{abstract}
Background: Multi-slice computed tomography (MSCT) can be useful before percutaneous mitral annuloplasty (PMA) procedures to visualize the relations between the mitral valve $(M V)$, left circumflex artery ( $L C x$ ) and coronary sinus (CS).

Methods and results: We performed a 64-slice MSCT in 196 patients (109 male; age $56.6 \pm$ \pm 11.4 ) with suspected coronary artery disease. A retrospective scan with ECG-gating was performed in each. In each case $3 D V R$ and $2 D$ MPR reconstructions were created. We used a subjective assessment of the quality of visualization to find the optimal phases of visualization for LCX, CS and both vessels together (relations). The quality of visualization were graded by 2 experts on 6-points scale. LCx was usually optimally visualized in the diastolic phases (70-80-90\%) - 126/196 (64.3\%). CS was usually optimally visualized in the systolic phases (30-40-50\%) - 177/196 (90.3\%). The optimal phase for parallel visualization of LCx/CS (to observe anatomical relations) was 70-80\% - 140/196 (71.4\%). Good quality visualization was obtained for both vessels: LCx: $3.6 \pm 1.4 / C S: 4.1 \pm 1.1$.

Conclusions: Reconstructions of parallel visualization of LCx/CS for PMA procedures to observe the relations between those vessels should be considered during diastole. In addition, independent reconstructions should also be performed optimized for the LCx and the CS. (Cardiol J 2012; 19, 5: 459-465)
\end{abstract}

Key words: percutaneous mitral annuloplasty, computed tomography, coronary vessels

\section{Introduction}

Mitral regurgitation is a disorder of the heart in which the mitral valve does not close properly. It can be caused by various mechanisms related to structural or functional abnormalities of the mitral valve or adjacent myocardium $[1,2]$. It is the second most common form of valvular heart disease after aortic stenosis with a prevalence of approxi- mately $2 \%$ of the population, affecting men and women equally. Echocardiography is the most frequent method used to confirm the diagnosis of mitral regurgitation. Color Doppler flow on the transthoracic echocardiogram will reveal a jet of blood flowing from the left ventricle into the left atrium during ventricular systole [3, 4]. In some cases, a transesophageal echocardiography may be needed to determine the severity of the regurgitation $[5,6]$.

Address for correspondence: Rafał Młynarski, MD, PhD, Upper Silesian Medical Center, Electrocardiology Department, ul. Ziołowa 45/47, 40-635 Katowice, Poland, fax: +48 32 2524098, e-mail: Rafal_Mlynarski@mp.pl

Received: 21.04.2012

Accepted: 28.05.2012 


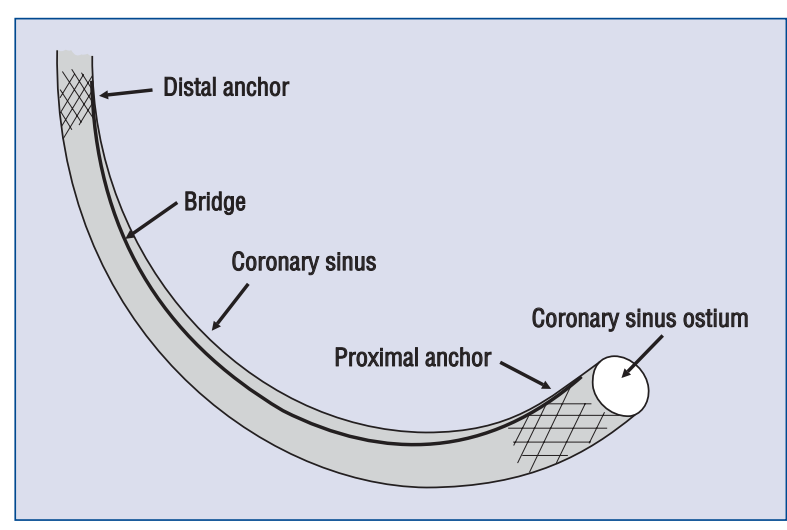

Figure 1. Scheme of the percutaneous mitral annuloplasty device based on MONARC device (Edwards Lifesinces, USA).

Treatment of mitral regurgitation depends on the severity of symptoms and whether there are associated signs of hemodynamic compromise.

Percutaneous mitral annuloplasty (PMA) is an alternative for patients with ischemic mitral regurgitation [7-9]. In this method the circumference of the mitral annulus is reduced in a antero-posterior diameter by using dedicated devices. Examples of such devices are: MONARC (Edwards Lifesinces, USA), CARILLON (Cardiac Dimensions, USA) and VIACOR PTMA (Viacvor, USA) [10, 11]. Scheme of MONARC is presented on the Figure 1. Devices are implanted percutaneously via the coronary sinus ostium from the right atrium and finally to the coronary sinus and great cardiac vein. It usually consist of two parts - the proximal part in the coronary sinus (CS) ostium and the distal part in the distal great cardiac vein. The two parts are connected by a "bridge" that reduces circumference of the mitral annulus.

In some anatomical variants, a close relationship between the left circumflex artery (LCx) and the CS can cause the LCx to be accidently occluded during placement of the device. This is main reason why multi slice computed tomography (MSCT) should be a standard procedure before PMA. This is to allow visualization of the relationships between the mitral valve (MV), the LCx and CS so that the risk of occluding the LCx can be minimized $[9,12]$. There are no recommendations how to visualize those structures during MSCT, despite the knowledge that the coronary arteries and coronary veins can be seen optimal in different phases of the cardiac cycle [13].

The purpose of this study was therefore to determine how optimally to visualize (and in which part of the cardiac cycle) the $\mathrm{LCx}$, the $\mathrm{CS}$ and their mutual relations during MSCT.

\section{Methods}

We performed a 64 -slice computed tomography (CT) in 196 patients (109 male, age $56.6 \pm 11.4$ years), for suspected coronary artery disease (CAD). We divided the patients into two groups: Group A - those without CAD and structural heart disease and Group B - those with CAD but without structural heart disease. Patients were excluded if they had atrial fibrillation, frequent premature heartbeats, renal insufficiency (serum creatinine $>1.2 \mathrm{mg} / \mathrm{dL}$ ), hyperthyreosis, known allergy to nonionic contrast agents and a previously implanted pacemaker with unipolar leads.

CT was performed using an Aquilion 64 scanner (Toshiba Medical Systems, Japan). Scanning with retrospective ECG-gating was performed during a breath-hold using 64 slices with a collimated slice thickness of $0.5 \mathrm{~mm}$. A breath-hold examination was performed to adjust the scanner settings. The helical pitch was 12.8 (best mode) and the rotation time was $0.4 \mathrm{~s}$. The tube voltage was strictly dependent on the patient's body mass index (BMI): for BMI $<23.9$ it was $120 \mathrm{kV}$ at $330 \mathrm{~mA}$, for $\mathrm{BMI}=24.0 \sim 29.9$ it was $135 \mathrm{kV}$ at $380 \mathrm{~mA}$ and for BMI > 30.0 it was $135 \mathrm{kV}$ at $430 \mathrm{~mA}$. We used a pre-selected region of interest (ROI) in the descending aorta. Triggering started at 180 Hounsfield units. On average, $100 \mathrm{~mL}$ of non-ionic contrast agent (Ioperamid, Ultravist 370, Schering, Germany) was given to each patient during the examination at an average rate of $4.5 \mathrm{~mL} / \mathrm{s}$. The contrast agent was given in three phases: $90 \mathrm{~mL}$ of contrast agent (average), then $24 \mathrm{~mL}$ of contrast agent followed by $16 \mathrm{~mL}$ of saline flush (60\%/40\%) and finally $30 \mathrm{~mL}$ of saline. The cut-off for heart rate (HR) was set at $65 \mathrm{bpm}$. If the HR was higher, metoprolol succinate (Betaloc, Astra Zeneca, Sweden) at a dosage of 5-10 mg was given intravenously, unless contraindicated. If the expected HR slowing did not occurred, the patient was excluded from the study. Sublingual nitroglycerin was not given before image acquisition.

Reconstructions of data were performed on Vitrea 2 workstations (Vital Images, USA; software version 3.9.0.0 and 5.1). In each case, we created ten (10) axial image series, as well as 3D volume rendering (VR) reconstructions, from $0 \%$ to $90 \%$ $\mathrm{R}-\mathrm{R}$ intervals (step 10\%) using a $2.0 \mathrm{~mm}$ slice thickness to reduce the large amount of data.

The optimal phases for visualization of the $\mathrm{LCx}$, $\mathrm{CS}$ and both vessels together (and their relation- 
Table 1. Scale of visualization used to the evaluation of left circumflex artery and coronary sinus.

\begin{tabular}{ll}
\hline Score & Description \\
\hline 0 & No vessel(s) present* \\
1 & Vessel, length less than $5 \mathrm{~mm}$, weakly contrasted and/or with a number of artifacts. \\
2 & Grade 1 means that a vessel is present but very poorly visualized \\
3 & Between score 1 and score 3 \\
4 & Bessel longer than $1 \mathrm{~cm}$, better contrasted. Sometimes areas not visible or artifacts occurred \\
5 & Vessel well contrasted, visible clearly on all the entire length of the vessel \\
\hline
\end{tabular}

*When the vein is not visualized in any of the phases it has grade 0 . However, this does not necessarily mean that the vein is absent - it could be e.g. very small and therefore lower than the resolution of computed tomography scanner.

Table 2. Average values (min, max, SD) of the main cardiac function parameters for the patients included.

\begin{tabular}{lcccc}
\hline & Average value & Min. value & Max. value & SD \\
\hline Ejection fraction [\%] & 62.75 & 8 & 86 & 9.29 \\
End-diastolic volume [mL] & 142.67 & 69 & 464 & 45.07 \\
End-systolic volume [mL] & 55.11 & 11 & 425 & 35.56 \\
Stroke volume [mL] & 86.92 & 39 & 151 & 20.28 \\
Cardiac output [L/min] & 5.38 & 1.8 & 13.9 & 1.63 \\
Myocardial mass [g] & 136.59 & 60 & 371 & 47.00 \\
Myocardial volume [mL] & 128.79 & 13 & 352 & 44.57 \\
Heart rate [bpm] & 61.16 & 20 & 83 & 8.39 \\
\hline
\end{tabular}

ship to one another) were judged on the quality of visualization. This graded by 2 experts trained in MSCT using a previously published 6-point scale (Table 1) [13, 14].

The local Ethics Committee approved the study protocol and all participants gave their written informed consent. The study protocol conformed to the version of the Helsinki Convention that was current at the time the study was designed.

\section{Results}

Hemodynamic characteristic of the patients is presented in the Table 2. Figure 2 shows examples of $3 \mathrm{D}$ visualizations of the $\mathrm{LCx}, \mathrm{CS}$ and their relationship during ten (10) phases of the cardiac cycle. Both vessels could usually be optimally visualized - for LCx the quality of visualization was $3.6 \pm 1.4$ and for the CS it was $4.1 \pm 1.1(\mathrm{p}=$ $=0.0009$ ). The quality of visualization for the $\mathrm{LCx}$ during diastole and for the CS during systole are presented in Table 3. We were not able to visualize the LCx in only 9 (4.6\%) patients. The influence of the R-R interval on the quality of reconstructions is given in Table 4 and Figure 3.

\section{Visualizing the LCx}

In 126 patients of 196 (64.3\%) the LCx was optimally visualized during the diastolic phases (70-80-90\%). In 69 (36.9\%) patients optimal image quality was obtained during the $80 \%$ phase, in $46(24.6 \%)$ patients during the $70 \%$ phase, and in 11 (5.9\%) patients during the $90 \%$ phase.

\section{Visualizing the CS}

In 177 patients of $196(90.3 \%)$ the optimal image quality for the CS was obtained during systole (30-40-50\%). In 118 (60.2\%) patients optimal image quality was obtained during the $40 \%$ phase, in $36(18.4 \%)$ patients during the $30 \%$ phase, and in 23 patients (11.7\%) during the $50 \%$ phase.

\section{Visualizing the $\mathrm{LCx}$ and $\mathrm{CS}$ in parallel}

The optimal phase for visualization both the LCx and the CS in parallel (to observe the anatomical relationship between the two vessels) was at $70-80 \%$. This was the case in 140 patients of $196(71.4 \%)$. In $76(40.6 \%)$ patients the optimal image quality was obtained during the $70 \%$ phase and in $64(34.2 \%)$ patients it was during the $80 \%$ phase. 


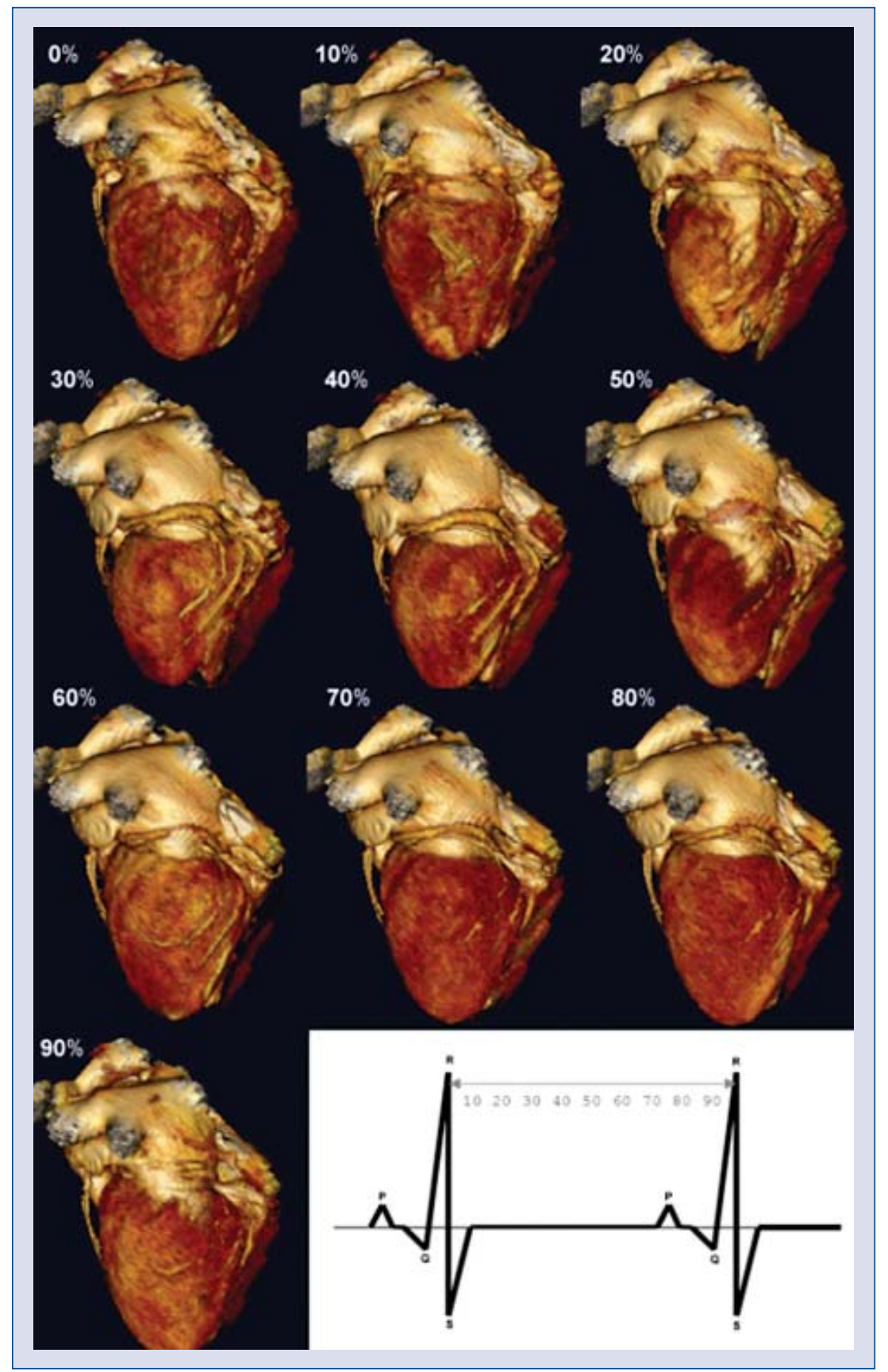

Figure 2. Influence of the phase of reconstruction on visualization of left circumflex artery and coronary sinus. Lateral view of the heart in 3D volume rendering reconstructions (MSCT).

The optimal phase for visualizing the analyzed vessels was highly reproducible (mean difference $-0.4 \%$; 95\% CI $-4.2-3.5 \%$, inter-rater agreement kappa $=$ $=0.95)$. Intra-observer agreement was also excellent (mean difference 0 , inter-rater agreement kappa $=1.0$ ).

Similarly, there was a good agreement between observers in the evaluation of the quality of the reconstructions. The mean values of the quality score $(\mathrm{LCx})$ were $3.10 \pm 1.59$ and $3.07 \pm 1.56$ and the mean difference -0.04 (95\% CI -1.07-1.00, inter-rater agreement kappa $=0.61)$. There were similar results in the repeated evaluation of the score by the same observer (mean difference 0.0 ; 95\% CI $-1.4-1.4$ and kappa $=0.66)$. 
Table 3. Quality of the visualization of the left circumflex artery (LCx) and coronary sinus (CS) system in score points for the three best visualized phases.

\begin{tabular}{|c|c|c|c|c|c|c|c|c|c|c|}
\hline & \multicolumn{2}{|c|}{ Score 5} & \multicolumn{2}{|c|}{ Score 4} & \multicolumn{2}{|c|}{ Score 3} & \multicolumn{2}{|c|}{ Score 2} & \multicolumn{2}{|c|}{ Score 1} \\
\hline Phase $70 \%$ & 17 & $9.66 \%$ & 12 & $6.8 \%$ & 10 & $5.68 \%$ & 1 & $0.57 \%$ & 0 & $0 \%$ \\
\hline Phase $80 \%$ & 25 & $14.20 \%$ & 23 & $13.1 \%$ & 7 & $3.79 \%$ & 7 & $3.79 \%$ & 1 & $0.57 \%$ \\
\hline Phase $90 \%$ & 5 & $2.84 \%$ & 3 & $1.71 \%$ & 2 & $1.14 \%$ & 0 & $0 \%$ & 1 & $0.57 \%$ \\
\hline \multicolumn{11}{|c|}{ Quality of visualization CS } \\
\hline Phase $30 \%$ & 19 & $10.79 \%$ & 9 & $5.11 \%$ & 2 & $1.14 \%$ & 3 & $1.71 \%$ & 3 & $1.71 \%$ \\
\hline Phase $40 \%$ & 61 & $34.66 \%$ & 31 & $17.61 \%$ & 18 & $10.23 \%$ & 6 & $3.41 \%$ & 2 & $1.14 \%$ \\
\hline Phase $50 \%$ & 8 & $4.45 \%$ & 5 & $2.84 \%$ & 9 & $5.11 \%$ & 1 & $1.71 \%$ & 0 & $0 \%$ \\
\hline
\end{tabular}

*Optimal phase to observe relations between LCx/CS

Table 4. Number of patients for whom optimal image quality in selected phases were achieved.

\begin{tabular}{|c|c|c|c|c|c|c|c|c|c|c|c|}
\hline & $\begin{array}{c}\text { Phase } \\
0 \%\end{array}$ & $\begin{array}{c}\text { Phase } \\
10 \%\end{array}$ & $\begin{array}{c}\text { Phase } \\
20 \%\end{array}$ & $\begin{array}{c}\text { Phase } \\
30 \%\end{array}$ & $\begin{array}{c}\text { Phase } \\
40 \%\end{array}$ & $\begin{array}{c}\text { Phase } \\
50 \%\end{array}$ & $\begin{array}{c}\text { Phase } \\
60 \%\end{array}$ & $\begin{array}{c}\text { Phase } \\
70 \%\end{array}$ & $\begin{array}{l}\text { Phase } \\
80 \%\end{array}$ & $\begin{array}{c}\text { Phase } \\
90 \%\end{array}$ & $\begin{array}{l}\text { Impossible } \\
\text { to evaluate }\end{array}$ \\
\hline LCx & 1 & 0 & 1 & 8 & 26 & 13 & 12 & 46 & 69 & 11 & 9 \\
\hline CS & 1 & 1 & 1 & 36 & 118 & 23 & 4 & 6 & 6 & 0 & 0 \\
\hline Optimal LCx/CS* & 2 & 0 & 1 & 2 & 24 & 7 & 10 & 76 & 64 & 1 & 9 \\
\hline
\end{tabular}

*Optimal phase to observe relations between LCx/CS; LCx — left circumflex artery; CS — and coronary sinus

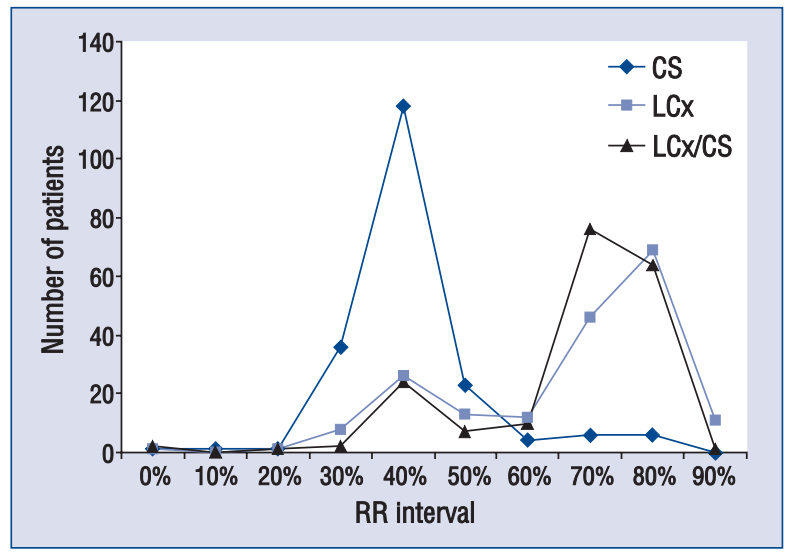

Figure 3. The distribution of the optimal phase of reconstruction of the left circumflex artery (LCX), coronary sinus (CS) and LCx/CS. Phases 70-80-90\% representing systole, phases $30-40-50 \%$ representing diastole.

\section{Discussion}

Percutaneous mitral annuloplasty devices was developed to treat patient with mitral ischemic regurgitation. Safety and usefulness were suggested in human studies like AMADEUS trial [15]. Potential benefits from using of PMA procedures are: to reshape the valvular dimensions, tighten the valve leaflets and what is more important to reduce mitral regurgitation. In the era of cardiac resynchronization, cannulation of coronary sinus usually is not a problem, but sometimes anatomy of heart vessels can be real challenge [16].

The anatomical relationship between the $\mathrm{LCx}$ and the CS is crucial for PMA. During animal studies early in the development this technique, LCx compression was reported as a serious complications. This emphasized the importance of being able to clearly see the anatomical relationship between the LCx, the CS and the MV.

Several studies have confirmed the considerable anatomical variability in the relative positions of the LCx, the CS and the MV. For example Maselli et al. [17], examined the hearts of 61 patients who had died of non-cardiological causes.

In the era of non-invasive visualization MSCT can play a vital role. One of the earliest studies was that of Tops et al. in 2007 [12]. The authors analyzed 105 consecutive patients referred for MSCT coronary angiography. Patients were divided into 3 groups according to the presence of CAD and heart failure. They concluded that the LCx, CS and mitral valve can be analyzed. This paper is relevant to our study as they used the same MSCT scanner with retrospective gating. The authors did not analyze the influence of the phase of the RR interval 
on their ability to visualize or the quality of visualization - their data sets were always reconstructed during $75 \%$ of the RR interval. The main purpose of our study was to analyzed the influence of the phase of the RR interval on the possibility of visualizing these key vessels and the quality of visualization. We found that the best visualization of the anatomical relations between those these vessels was at $70-80 \%$ of the RR. This is comparable with phase used by Tops et al. [12]. Nevertheless in almost $30 \%$ of patients better visualization was obtained in different phases.

Plass et al. [18] carried out a retrospective analysis of 50 patients who had MSCT before minimally invasive valve surgery. They performed 64 slice CT (GE Healthcare) in all the patients. Like Tops et al. [12] they reconstructed their data at the $75 \%$ phase. Their results confirmed that MSCT allows the exact depiction and measurement of the CS, coronary arteries and the MV with the surrounding tissue.

Choure et al. [19] used MSCT to examine the hearts of 25 patients with normal mitral function and 14 with severe mitral regurgitation using a 16 slice scanner (Philips Medical Systems) with retrospective gating. They did not give exact information about the phase of the RR interval they used. Instead, they reported that reconstructions were created at the desired phases during the cardiac cycle. Exact information about RR interval is not available in this publication - the only information is that reconstructions were created at desired phases during the cardiac cycle. Nevertheless they confirmed that there is significant variability in the relative anatomical positions of the $\mathrm{LCx}, \mathrm{CS}$, and MV annulus.

Despite the differences between these published studies, there is agreement about the usefulness of MSCT especially in identifying patients in whom this method could lead to serious complications, thus helping in the precise pre-selection of patients and planning the procedure individually for qualified patients.

Earlier publications including studies from our unit have shown that the phase of the RR interval at which reconstructions are performed is an important factor affecting the quality of visualization $[13,14,20]$. This is especially important when vessels other then coronary arteries need to be analyzed. Arteries are optimally visualized during diastole (70-80-90\% of the RR interval). This is the basis for MSCT with prospective gating where the best quality is only during diastolic phases. In contrast, in retrospective scanners all phases are scanned and any phase can be reconstructed with full quality. However the major disadvantage of retrospective reconstruction is that the patient is exposed to high doses of radiation.

It has been reported that coronary veins are seen optimally during systole (30-40-50\% of the $\mathrm{RR}$ interval). However there is still some uncertainty about how best to visualize both coronary arteries and coronary veins. The same problem is met during pre-visualization of the coronary venous system before cardiac resynchronization therapy (CRT). During these procedures the coronary arteries are visualized as a basic procedure, and the coronary veins are analyzed as a secondary procedure to evaluate their anatomy, in particular the coronary sinus ostium and target veins for CRT. This poses much the same challenges as percutaneus mitral annuloplasty procedures. The next step in the development of PMA connection with CRT. The basis is that in patients with heart failure (potential candidates for CRT) mitral regurgitation often coexists with heart failure. In such cases additional visualization of coronary venous system should be added [13].

There are some reports existing, where CRT devices were implanted in the group of patients after PMA procedures. Hoppe et al. [21] reported group of three patients from AMADEUS trial underwent CRT after 6 months of follow up. This small study proofed the feasibility of positioning a left ventricle lead after permanent implantation of PMA device (CARILLON).

Pre-visualization of the vessels in diastole (coronary arteries) and in systole (coronary veins) can be used in the future to evaluate target veins before cardiac resynchronization.

\section{Limitations of the study}

Most of the limitations are related to the MSCT as a method. The dose of radiation is substantial, as well as amount of contrast agent. However the risk of iatrogenic myocardial infarction due to in accidental occlusion of the LCx during PMA is probably higher than the limitation inherent in MSCT.

Our study is about methodology, our aim being only to evaluate how to optimally visualize the coronary vessels and their mutual relations on MSCT. The results have only practical implications for performing MSCT, and not for practical performing of PMA procedures.

\section{Conclusions}

It is possible to visualize the CS and $\mathrm{LCx}$ in relation to the mitral valve during MSCT. Recon- 
structions of parallel visualization of both the $\mathrm{LCx}$ and the CS to determine the relations between these vessels before PMA procedures should be considered during diastolic phase. Additional independent reconstruction optimal for LCx (mostly diastolic) as well as CS (mostly systolic) should also be performed.

\section{Acknowledgments}

The authors are indebted to the radiology technologists for their technical support.

\section{Conflict of interest: none declared}

\section{References}

1. Gillam LD. Is it time to update the definition of functional mitral regurgitation? structural changes in the mitral leaflets with left ventricular dysfunction. Circulation, 2008; 118: 797-799.

2. Enriquez-Sarano M, Akins CW, Vahanian A. Mitral regurgitation. Lancet, 2009; 373: 1382-1394.

3. Zakkar M, Patni R, Punjabi PP. Mitral valve regurgitation and 3D echocardiography. Future Cardiol, 2010; 6: 231-242.

4. Miller AP, Nanda NC. Live/real-time three-dimensional transthoracic assessment of mitral regurgitation and mitral valve prolapse. Cardiol Clin, 2007; 25: 319-325.

5. Khanna D, Miller AP, Nanda NC, Ahmed S, Lloyd SG. Transthoracic and transesophageal echocardiographic assessment of mitral regurgitation severity: Usefulness of qualitative and semiquantitative techniques. Echocardiography, 2005; 22: 748-769.

6. Vahanian A, Baumgartner H, Bax J et al.; Task Force on the Management of Valvular Hearth Disease of the European Society of Cardiology; ESC Committee for Practice Guidelines. Guidelines on the management of valvular heart disease. The Task Force on the Management of Valvular Heart Disease of the European Society of Cardiology. Eur Heart J, 2007; 28: 230-268.

7. Masson JB, Webb JG. Percutaneous mitral annuloplasty. Coron Artery Dis, 2009; 20: 183-188.

8. Johnston DR, Gillinov AM, Blackstone EH et al. Surgical repair of posterior mitral valve prolapse: Implications for guidelines and percutaneous repair. Ann Thorac Surg, 2010; 89: 1385-1394.

9. Gopal A, Shah A, Shareghi S et al. The role of cardiovascular computed tomographic angiography for coronary sinus mitral annuloplasty. J Invasive Cardiol, 2010; 22: 67-73.
10. Marsan NA, Bax JJ. Non-invasive imaging in percutaneous mitral valve procedures. EuroIntervention, 2010; 6 (Suppl. G): G28-G37.

11. Sack S. Percutaneous mitral annuloplasty with the VIACOR coronary sinus system for the treatment of functional mitral regurgitation in heart failure patients. Development and results. Herz, 2009; 34: 468-476.

12. Tops LF, Van de Veire NR, Schuijf JD et al. Noninvasive evaluation of coronary sinus anatomy and its relation to the mitral valve annulus: Implications for percutaneous mitral annuloplasty. Circulation, 2007; 115: 1426-1432.

13. Mlynarski R, Sosnowski M, Wlodyka A, Chromik K, Kargul W, Tendera M. Optimal image reconstruction intervals for noninvasive visualization of the cardiac venous system with a 64 -slice computed tomography. Int J Cardiovasc Imag, 2009; 25: 635-641.

14. Mlynarska A, Mlynarski R, Kargul W, Sosnowski M. Quality of visualization of coronary venous system in 64-slice computed tomography. Cardiol J, 2011; 18: 146-150.

15. Siminiak T, Hoppe UC, Schofer J et al. Effectiveness and safety of percutaneous coronary sinus-based mitral valve repair in patients with dilated cardiomyopathy (from the AMADEUS trial). Am J Cardiol, 2009; 104: 565-570.

16. Luedorff G, Grove R, Kranig W, Thale J. Different venous angioplasty manoeuvres for successful implantation of CRT devices. Clin Res Cardiol, 2009; 98: 159-164.

17. Maselli D, Guarracino F, Chiaramonti F, Mangia F, Borelli G, Minzioni G. Percutaneous mitral annuloplasty: An anatomic study of human coronary sinus and its relation with mitral valve annulus and coronary arteries. Circulation, 2006; 114: 377-380.

18. Plass A, Valenta I, Gaemperli O et al. Assessment of coronary sinus anatomy between normal and insufficient mitral valves by multi-slice computertomography for mitral annuloplasty device implantation. Eur J Cardiothorac Surg, 2008; 33: 583-589.

19. Choure AJ, Garcia MJ, Hesse B et al. In vivo analysis of the anatomical relationship of coronary sinus to mitral annulus and left circumflex coronary artery using cardiac multidetector computed tomography: Implications for percutaneous coronary sinus mitral annuloplasty. J Am Coll Cardiol, 2006; 48: 1938-1945.

20. Mlynarski R, Sosnowski M, Wlodyka A, Kargul W, Tendera M. A user-friendly method of cardiac venous system visualization in 64-slice computed tomography. Pacing Clin Electrophysiol, 2009; 32: 323-329.

21. Hoppe UC, Brandt MC, Degen H et al. Percutaneous mitral annuloplasty device leaves free access to cardiac veins for resynchronization therapy. Catheter Cardiovasc Interv, 2009; 74: 506-511. 